\title{
CONVEX BODIES WITH SIMILAR PROJECTIONS
}

\author{
R. J. GARDNER AND A. VOLČIČ \\ (Communicated by Palle E. T. Jorgensen)
}

\begin{abstract}
By examining an example constructed by Petty and McKinney, we show that there are pairs of centered and coaxial bodies of revolution in $\mathbb{E}^{d}$, $d \geq 3$, whose projections onto each two-dimensional subspace are similar, but which are not themselves even affinely equivalent.
\end{abstract}

\section{INTRODUCTION}

In [H], Hadwiger proved the following theorem: If $K_{1}$ and $K_{2}$ are convex bodies in $\mathbb{E}^{d}, 2 \leq k \leq d-1$, and the projections of $K_{1}$ and $K_{2}$ onto each $k$-dimensional subspace are directly homothetic, then $K_{1}$ and $K_{2}$ must also be directly homothetic. (The case $d=3$ was first published by Süss in [S] and Nakajima in [N].) Later, in [R], Rogers showed that the result remains true when projections are replaced by sections through some common interior point of $K_{1}$ and $K_{2}$. The two theorems raised questions which led to significant developments in the study of projections and sections of convex bodies. The result on sections began a string of papers on the so-called False Center Conjecture, culminating in the powerful theorem of Burton and Mani in [BM]. Also, Petty and McKinney [PM] found an example to show that certain generalizations of the two theorems in $[R]$ are not possible.

The Petty-McKinney example demonstrates that the hypotheses in the above theorems that projections (or sections) are directly homothetic cannot be replaced by the assumption of similarity. There is certainly one clear difference between direct homothety and similarity with respect to projections; projections of directly homothetic convex bodies are directly homothetic, while a simplex and a rotation of it will generally not have similar projections. Nevertheless, the Petty-McKinney example is extremely surprising and deserves to be better known. In [PM] it is shown that there are pairs $K_{1}, K_{2}$ of centered (centrally symmetric with center at the origin) convex bodies in $\mathbb{E}^{d}, d \geq 3$, such that for each two-dimensional subspace $S$ the projection $K_{1} \mid S$ of $K_{1}$ onto $S$ is directly homothetic to a rotation of $K_{2} \mid S$ by $\pi / 2$ about the origin, yet $K_{1}$ and

Received by the editors September 9, 1992.

1991 Mathematics Subject Classification. Primary 52A20.

Key words and phrases. Convex body, projection, section, direct homothety, similarity, affine equivalence.

The first author was supported in part by NSF Grant DMS 9201508. The second author was supported in part by Italian Research Council (CNR) Grant 91.01347.09. 
$K_{2}$ are not directly homothetic. Moreover, Theorem 3.1 of [PM] characterizes all pairs $K_{1}, K_{2}$ with these properties. Corresponding examples for sections instead of projections follow immediately using polar duality.

It is worth noting that the Petty-McKinney example also serves to provide contrast to the famous uniqueness theorems of Alexandrov and Funk. In [A] and $[\mathrm{F}]$ it is proved that if $1 \leq k \leq d-1$ and two centered convex bodies in $\mathbb{E}^{d}$ are such that their projections onto (or sections by, respectively) each $k$-dimensional subspace have the same $k$-dimensional volume, then they must be equal. In particular, they must be equal if their projections or sections are congruent.

Like Roger's theorem, the Petty-McKinney example also raises some natural questions, and it is the purpose of this note to answer some of these. We show that although it is possible for pairs $K_{1}, K_{2}$ in the Petty-McKinney example to be similar (but not directly homothetic), there are pairs which are not even affinely equivalent. We also prove that such pairs are affinely equivalent if and only if they are similar and characterize when this can occur. It follows that direct homothety in Hadwiger's theorem cannot be replaced throughout by either similarity or affine equivalence. Again, polar duality yields the corresponding results for sections.

\section{DEFINITIONS AND PRELIMINARIES}

We denote $d$-dimensional Euclidean space by $\mathbb{E}^{d}$ and its unit sphere and origin by $S^{d-1}$ and $\underline{o}$, respectively. If $S$ is a subspace, then $E \mid S$ is the orthogonal projection of the set $E$ onto $S$.

A convex body is a compact convex set with nonempty interior. We say a convex body is centered if it is centrally symmetric, with center $\underline{o}$. If $K$ is a convex body, we write $h_{K}$ for its support function (see, for example, [BF, §15]). Suppose $A$ is a nonsingular affine transformation of $\mathbb{E}^{d}$ with the transpose denoted by $A^{T}$. Then it follows easily from the definition of $h_{K}$ that

$$
h_{A K}(x)=h_{K}\left(A^{T} x\right) \text {, }
$$

for all $x \in \mathbb{E}^{d}$.

Consider pairs $K_{1}, K_{2}$ of convex bodies defined as follows. The support function $h_{K_{1}}$ of $K_{1}$ is defined for nonzero $x \in \mathbb{E}^{d}$ by

$$
h_{K_{1}}(x)=\|x\| \exp \left(\frac{x^{T} C x}{\|x\|^{2}}\right),
$$

where $C$ is any real symmetric matrix of order $d$, with eigenvalues $c_{1}, \ldots, c_{d}$ satisfying the condition $\max \left|c_{i}-c_{j}\right| \leq \frac{1}{2}$. The support function $h_{K_{2}}$ of $K_{2}$ is defined similarly, where the matrix $C$ is replaced by $-C$ (whose eigenvalues satisfy the same condition). The authors of [PM] show that these are precisely the pairs of centered convex bodies in $\mathbb{E}^{d}, d \geq 3$, such that the projection of one onto each two-dimensional subspace is directly homothetic to a rotation by $\pi / 2$ about the origin of the projection onto the same subspace of the other.

\section{Results}

Theorem. The convex bodies $K_{1}, K_{2}$ of the Petty-McKinney example are affinely equivalent if and only if they are similar, and this occurs if and only if there is a 
constant a such that the eigenvalues $c_{i}$ of the matrix $C$, arranged so that they increase with $i$, satisfy

$$
c_{i}+c_{d+1-i}=a
$$

for $i=1, \ldots, d$.

Proof. Let $K_{1}, K_{2}$ be a pair of convex bodies in $\mathbb{E}^{d}, d \geq 3$, with support functions defined as in $\S 2$. By applying an orthogonal transformation, if necessary, we may assume $C$ to be a diagonal matrix such that $c_{1} \leq c_{2} \leq \cdots \leq c_{d}$.

Suppose $A K_{1}=K_{2}$, where $A$ is a nonsingular affine map. We shall prove that $K_{1}$ and $K_{2}$ are similar and (1) holds. Since $K_{i}$ is centered, $i=1,2, A$ must actually be a linear map. For, let $[x,-x]$ be a chord of $K_{1}$ containing $\underline{o}$, and therefore bisected by $\underline{o}$. Then $[A x,-A x]$ is a chord of $K_{2}$ which is bisected by $A \underline{\underline{O}}$, so $K_{2}$ is centrally symmetric about $A \underline{o}$. But $\underline{O}$ is the center of $K_{2}$, so $A \underline{o}=\underline{o}$.

We have $\bar{h}_{K_{2}}(x)=h_{A K_{1}}(x)=h_{K_{1}}\left(A^{T} x\right)$, from which we obtain

$$
\left\|A^{T} u\right\|^{2}=\exp \left(-2 \sum_{i=1}^{d} \frac{c_{i}\left(A^{T} u\right)_{i}^{2}}{\left\|A^{T} u\right\|^{2}}-2 \sum_{i=1}^{d} c_{i} u_{i}^{2}\right),
$$

for all $u \in S^{d-1}$. Let us first set $u_{1}=2 z /\left(1+z^{2}\right), u_{2}=\left(1-z^{2}\right) /\left(1+z^{2}\right)$, and $u_{i}=0$ for $i=3, \ldots, d$. We claim that both sides of (2) are then constant.

The substitution yields an equation of the form $p(z) /\left(1+z^{2}\right)^{2}=e^{f(z)}$, where $p(z)$ is a polynomial of degree at most four. We rewrite this in the form

$$
p(z)=\left(1+z^{2}\right)^{2} e^{f(z)},
$$

which then holds for all real $z$. Further, $f(z)$ is a rational function whose denominator is nonzero for each real $z$. Therefore, both sides of the equation represent functions which are analytic in a domain in the complex plane $\mathbb{C}$ containing the real axis. Since $p(z)$ is a polynomial, we may take its domain to be the whole of $\mathbb{C}$, and then a standard uniqueness theorem (see, for example, [C, Theorem 1, p. 261]) implies that the last equation holds for all $z \in \mathbb{C}$. The exponential function has no zeros in $\mathbb{C}$, so the only zeros of the right-hand side are double zeros at $z= \pm i$. These must then be precisely the zeros of the left-hand side, implying that $p(z)$ is a constant multiple of $\left(1+z^{2}\right)^{2}$ and hence that $e^{f(z)}$ is constant. Therefore, both sides of (2) are constant, under the assumption that $u_{i}=0$ for $i=3, \ldots, d$.

This implies that the first two columns of the matrix $A^{T}$ are orthogonal and the sum of the squares of the entries in each of these columns is the same.

The same conclusion can now be drawn for any pair of columns by replacing $u_{1}$ and $u_{2}$ by the appropriate pair of coordinates of $u$. It follows that $A^{T}$ is an orthogonal matrix $W=\left(w_{i j}\right)$ multiplied by a constant, $b^{-1}$ say. (This means that $A$ must be a similarity.) Substituting in (2) and using $\sum_{i=1}^{d} u_{i}^{2}=1$, we obtain

$$
\sum_{i=1}^{d} c_{i}(W u)_{i}^{2}+\sum_{i=1}^{d} c_{i} u_{i}^{2}=\log |b|
$$


Comparing coefficients, we see that

$$
\sum_{i=1}^{d} c_{i} w_{i j} w_{i k}=0,
$$

while the orthogonality of $W$ yields

$$
\sum_{i=1}^{d} w_{i j} w_{i k}=0
$$

whenever $1 \leq j \neq k \leq d$. Let $y_{j}, z_{j}$ denote the vectors whose $i$ th coordinates are $c_{i} w_{i j}, w_{i j}$, respectively. The last two equations imply that both $y_{j}$ and $z_{j}$ are orthogonal to $z_{k}$ for $k \neq j$. The vectors $z_{k}$ are just the columns of the matrix $W$, so for each $j$ the $z_{k}$ 's such that $k \neq j$ span a $(d-1)$-dimensional subspace. It follows that $y_{j}=t_{j} z_{j}$ for some real $t_{j}$ and all $j$. This means that

$$
c_{i} w_{i j}=t_{j} w_{i j}
$$

for all $i$ and $j$.

For each $m, 1 \leq m \leq d$, define $I_{m}=\left\{i: c_{i}=c_{m}\right\}$, and $J_{m}=\left\{j: w_{i j} \neq\right.$ 0 for some $\left.i \in I_{m}\right\}$. Then if $j \notin J_{m}$, we have $w_{i j}=0$ for all $i \in I_{m}$. Since $J_{m} \neq \varnothing$, by the orthogonality of $W$, we can choose a $p \in J_{m}$. Suppose that $w_{i p} \neq 0$ for some $i \notin I_{m}$. Then by (4) $c_{i} w_{i p}=t_{p} w_{i p}$. Also, there is an $i^{\prime} \in I_{m}$ with $w_{i^{\prime} p} \neq 0$. Using (4) again, $c_{i^{\prime}} w_{i^{\prime} p}=t_{p} w_{i^{\prime} p}$, which gives $c_{i}=c_{i^{\prime}}=c_{m}$, a contradiction. Therefore, $w_{i p}=0$ for each $i \notin I_{m}$. Let $e_{p}$ denote the unit vector in the $p$ th coordinate direction. Then

$$
\sum_{i \notin I_{m}}\left(W e_{p}\right)_{i}^{2}=0
$$

so that

$$
\sum_{i \in I_{m}}\left(W e_{p}\right)_{i}^{2}=\sum_{i=1}^{d}\left(W e_{p}\right)_{i}^{2}=1
$$

Substituting $u=e_{p}$ in the left-hand side of (3) then yields

$$
\sum_{i \in I_{m}} c_{i}\left(W e_{p}\right)_{i}^{2}+c_{p}=c_{m}+c_{p}
$$

Consequently, for each $m, 1 \leq m \leq d$, there is a $p$ with $c_{m}+c_{p}=\log |b|$. The fact that the eigenvalues $c_{i}$ increase with $i$ now forces

$$
c_{i}+c_{d+1-i}=\log |b| \text {, }
$$

for $i=1, \ldots, d$, which means that (1) holds.

Suppose now that (1) is true, where $a=\log |b|$. Again, applying an orthogonal transformation, if necessary, we may assume that the matrix $C$ is diagonal. Then, for $u \in S^{d-1}$,

$$
\begin{aligned}
h_{K_{1}}(u) & =\exp \left(\sum_{i=1}^{d} c_{i} u_{i}^{2}\right)=\exp \left(\log |b|-\sum_{i=1}^{d} c_{d+1-i} u_{i}^{2}\right) \\
& =|b| \exp \left(-\sum_{i=1}^{d} c_{i} u_{d+1-i}^{2}\right)=|b| h_{K_{2}}(W u),
\end{aligned}
$$


where $W$ is the orthogonal matrix which interchanges the $i$ th and $(d+1-i)$ th coordinate axes for $i=1, \ldots, d$. So $K_{1}$ and $K_{2}$ are similar.

Corollary. For $d \geq 3$, there are centered, coaxial convex bodies of revolution $K_{1}$ and $K_{2}$ in $\mathbb{E}^{d}$ with the property that, for each two-dimensional subspace $S$, $K_{1} \mid S$ and $K_{2} \mid S$ are similar but $K_{1}$ and $K_{2}$ are not affinely equivalent.

Proof. Let the convex bodies $K_{1}$ and $K_{2}$ be as in the Petty-McKinney example, with $C$ the diagonal matrix with eigenvalues $c_{1}=\frac{1}{2}$ and $c_{i}=1$ for $i=$ $2, \ldots, d$. The corollary follows immediately, since equation (1) fails.

It is easy to see that the convex bodies $K_{1}$ and $K_{2}$ of the Petty-McKinney example are directly homothetic if and only if the eigenvalues $c_{i}$ of the matrix $C$ are all equal, that is, precisely when both bodies are centered balls. It is therefore possible for $K_{1}$ and $K_{2}$ to be similar but not directly homothetic. For example, take $d=3$ and $C$ to be the diagonal matrix with diagonal entries $\frac{1}{4}, \frac{1}{2}$, and $\frac{3}{4}$.

\section{FURTHER QUESTIONS}

The results above suggest the following natural problems.

Question 4.1. Suppose $2<k \leq d-1$ and $K_{1}$ and $K_{2}$ are centered convex bodies in $\mathbb{E}^{d}$ with $K_{1} \mid S$ similar to $K_{2} \mid S$, for every $k$-dimensional subspace $S$. Is $K_{1}$ similar to $K_{2}$ ?

Question 4.2. Suppose $2 \leq k \leq d-1$ and $K_{1}$ and $K_{2}$ are arbitrary convex bodies in $\mathbb{E}^{d}$ such that $K_{1} \mid S$ is congruent to $K_{2} \mid S$, for every $k$-dimensional subspace $S$. Is $K_{1}$ a translate of $\pm K_{2}$ ?

In $\left[G_{1}\right]$ and $\left[G_{2}\right]$, Golubyatnikov proves that the answer to Question 4.2 is positive when $k=2$ and none of the projections $K_{1} \mid S$ and $K_{2} \mid S$ has an extra symmetry with respect to rotations. In fact, in $\left[\mathrm{G}_{2}\right.$, Theorem 6$]$ it is shown that if $K_{1}$ and $K_{2}$ are convex bodies in $\mathbb{E}^{d}$ such that $K_{1} \mid S$ is similar to $K_{2} \mid S$, for every 2-dimensional subspace $S$, and none of the projections $K_{1} \mid S$ and $K_{2} \mid S$ has an extra symmetry, then $K_{1}$ is homothetic to $\pm K_{2}$. This still leaves open the following question.

Question 4.3. Suppose $K_{1}$ and $K_{2}$ are centered convex bodies in $\mathbb{E}^{d}$ such that $K_{1} \mid S$ is similar to $K_{2} \mid S$, for every two-dimensional subspace $S$. Must $K_{1}$ and $K_{2}$ be a pair as in the Petty-McKinney example?

\section{REFERENCES}

[A] A. D. Alexandrov, On the theory of mixed volumes of convex bodies, II. New inequalities between mixed volumes and their applications, Mat. Sb. 2 (1937), 1205-1238. (Russian)

[BM] G. R. Burton and P. Mani, A characterisation of the ellipsoid in terms of concurrent sections, Comment. Math. Helv. 53 (1978), 485-507.

[BF] T. Bonnesen and W. Fenchel, Theory of convex bodies, BCS Associates, Moscow, Idaho, 1987.

[C] R. V. Churchill, Complex variables and applications, McGraw-Hill, New York, 1960.

[F] P. Funk, Über Flächen mit lauter geschlossenen geodätischen Linien, Math. Ann. 74 (1913), 278-300.

$\left[\mathrm{G}_{1}\right]$ V. P. Golubyatnikov, Unique determination of visible bodies from their projections, Siberian Math. J. 29 (1988), 761-764. 
$\left[\mathrm{G}_{2}\right]+$, On unique recoverability of convex and visible compacta from their projections, Math. USSR Sb. 73 (1991), 1-10.

[H] H. Hadwiger, Seitenrisse konvexer Körper und Homothetie, Elem. Math. 18 (1963), 97-98.

[N] S. Nakajima, Eine Kennzeichnung homothetische Eiflächen, Tôhoku Math. J. 35 (1932), 285-286.

[PM] C. M. Petty and J. R. McKinney, Convex bodies with circumscribing boxes of constant volume, Portugal. Math. 44 (1987), 447-455.

[R] C. A. Rogers, Sections and projections of convex bodies, Portugal. Math. 24 (1965), 99-103.

[S] W. Süss, Zusammensetzung von Eikörpern und homothetische Eiflächen, Tôhoku Math. J. 35 (1932), 47-50.

Department of Mathematics, Western Washington University, Bellingham, WASHINGTON 98225-9063

E-mail address: gardner@baker.math.wwu.edu

Dipartimento di Scienze Matematiche, Universitá degli Studi di Trieste, 34100 TriESTE, ITALY

E-mail address: volcic@univ.trieste.it 